\title{
PROPORTIONALLY MODULAR DIOPHANTINE INEQUALITIES AND THE STERN-BROCOT TREE
}

\author{
M. BULLEJOS AND J. C. ROSALES
}

\begin{abstract}
Given positive integers $a, b$ and $c$ to compute a generating system for the numerical semigroup whose elements are all positive integer solutions of the inequality $a x \bmod b \leq c x$ is equivalent to computing a Bézout sequence connecting two reduced fractions. We prove that a proper Bézout sequence is completely determined by its ends and we give an algorithm to compute the unique proper Bézout sequence connecting two reduced fractions. We also relate Bézout sequences with paths in the Stern-Brocot tree and use this tree to compute the minimal positive integer solution of the above inequality.
\end{abstract}

\section{INTRODUCTION}

A proportionally modular diophantine inequality is an inequality of the form

$$
a x \bmod b \leq c x,
$$

with $a, b$ and $c$ positive integers. A nonnegative integer $x$ is a solution of such an inequality if the remainder of the division of $a x$ by $b$ is less than or equal to $c x$. The set $S$ of nonnegative integers which are solutions of (10) is a numerical semigroup (see [5]), that is, $S$ is a subset of the set $\mathbb{N}$, of nonnegative integers, which is closed under addition and such that $0 \in S$ and its complementary $\mathbb{N} \backslash S$ is finite. Not every numerical semigroup is the set of solutions of an inequality like (11) (see also [5]). Those fulfilling this condition are called proportionally modular numerical semigroups.

In [5] proportionally modular numerical semigroups are characterized. Let us introduce some notation that is used there to give such a characterization. The set of nonnegative reals will be denoted by $\mathbb{R}_{0}^{+}$. Given a subset $A \subseteq \mathbb{R}_{0}^{+}$we will write $\langle A\rangle$ for the submonoid of $\left(\mathbb{R}_{0}^{+},+\right)$generated by $A$. The elements in $\langle A\rangle$ are then linear combinations of the elements in $A$ with nonnegative integer coefficients.

Given reduced fractions $\frac{r}{s}<\frac{u}{v}$, with positive numerators and denominators, the semigroup $S\left(\left[\frac{r}{s}, \frac{u}{v}\right]\right)$ is the numerical semigroup associated to the closed interval $\left[\frac{r}{s}, \frac{u}{v}\right]$. That is,

$$
S\left(\left[\frac{r}{s}, \frac{u}{v}\right]\right)=\left\langle\left[\frac{r}{s}, \frac{u}{v}\right]\right\rangle \cap \mathbb{N} .
$$

Received by the editor January 3, 2008 and, in revised form, April 4, 2008.

2000 Mathematics Subject Classification. Primary 11D75, $20 \mathrm{M} 14$.

Key words and phrases. Diophantine inequation, numerical semigroup, Stern-Brocot tree.

This work was partially supported by research projects MTM2005-03227 and MTM2007-62346. 
Then, it is proved in [5] that the proportionally modular numerical semigroup $S$ coming from an inequality like (11) is

$$
S=S\left(\left[\frac{b}{a}, \frac{b}{a-c}\right]\right),
$$

and conversely, any numerical semigroup $S\left(\left[\frac{r}{s}, \frac{u}{v}\right]\right)$ is the set of solutions of an inequality like (11). In this case $a=u s, b=r u$ and $c=u s-r v$.

Thus, the problem of solving a proportionally modular diophantine inequality is reduced to that of finding a system of generators of a proportionally modular numerical semigroup, which is always in the form $S\left(\left[\frac{r}{s}, \frac{u}{v}\right]\right)$. This problem is reduced further (see [6]) to that of finding a Bézout sequence connecting the reduced fractions $\frac{r}{s}$ and $\frac{u}{v}$.

A Bézout sequence is a sequence of reduced fractions

$$
\frac{a_{1}}{b_{1}}<\frac{a_{2}}{b_{2}}<\cdots<\frac{a_{k}}{b_{k}}
$$

with positive numerators and denominators, which, moreover, satisfies the identities

$$
a_{i+1} b_{i}-a_{i} b_{i+1}=1, i \in\{1, \ldots, k-1\} .
$$

The number of terms, $k$, is the length of the sequence and the fractions $\frac{a_{1}}{b_{1}}$ and $\frac{a_{k}}{b_{k}}$ are respectively the left and right ends of the sequence. In [6] it is proved that any two reduced fractions with positive numerators and denominators can be connected by a Bézout sequence and, moreover, if (2) is a sequence connecting $\frac{r}{s}$ and $\frac{u}{v}$, that is $\frac{r}{s}=\frac{a_{1}}{b_{1}}$ and $\frac{u}{v}=\frac{a_{k}}{b_{k}}$, then

$$
S\left(\left[\frac{r}{s}, \frac{u}{v}\right]\right)=\left\langle r=a_{1}, a_{2}, \ldots, a_{k}=u\right\rangle .
$$

Hence the problem of solving an inequality like (1) is reduced to that of finding a Bézout sequence connecting two reduced fractions. We devote this paper to giving algorithmic methods to find such sequences.

A Bézout sequence with ends $\frac{r}{s}$ and $\frac{u}{v}$ is called proper if it has no proper subsequences with ends $\frac{r}{s}$ and $\frac{u}{v}$ that are Bézout sequences. It is clear that any Bézout sequence can be reduced (by dropping terms) to a proper one. We say that the sequence (2) has decreasing numerators if $a_{1} \geq a_{2} \geq \cdots \geq a_{k}$ and that it has increasing denominators if $b_{1} \leq b_{2} \leq \cdots \leq b_{k}$. In Section 2 we see that any proper Bézout sequence can be obtained by joining a Bézout sequence with decreasing numerators to a Bézout sequence with increasing denominators. We also prove that a Bézout sequence with decreasing numerators is completely determined by its left end and its length, and dually a Bézout sequence with increasing denominators is determined by its right end and its length. We then deduce that any two reduced fractions are connected by a unique proper Bézout sequence. In Section 3 we use the study of proper Bézout sequences done in Section 2 to give an algorithm (with similar complexity to Euclides' algorithm for the calculus of greater common divisors) for the computation of the unique proper Bézout sequence connecting two given reduced fractions.

In Section 4 we give a characterization, in terms of Bézout sequences, of when two fractions in the Stern-Brocot tree are one descendent of the other. This characterization will allow us to give another method, based on the calculus of paths in the Stern-Brocot tree, to compute the proper Bézout sequence connecting two reduced fractions. 
The multiplicity of a numerical semigroup $S$ is the smallest positive integer that is in $S$ and the Frobenius number of $S$ is the greatest integer that is not in $S$. The Frobenius problem consists of giving a formula to compute the Frobenius number of $S$ in terms of its generators. If $S=\left\langle n_{1}, n_{2}\right\rangle$, then the Frobenius number of $S$ is $n_{1} n_{2}-n_{1}-n_{2}$ (see [8]), but for numerical semigroups generated by more than two elements the Frobenius problem is still open (see [4); there is no hope for a similar formula when $n \geq 3$ since the problem of computing the Frobenius number is computationally difficult 3 .

In 7 an algorithm is given (with similar complexity to the Euclidean algorithm) to compute the multiplicity of a proportionally modular numerical semigroup and it is also proved there that if we have a formula to compute the multiplicity of the proportionally modular numerical semigroup $S\left(\left[\frac{r}{s}, \frac{u}{v}\right]\right)$, in terms of $\frac{r}{s}$ and $\frac{u}{v}$, the Frobenius problem will be solved for numerical semigroups that can be generated by three elements. In Section 5 we relate the multiplicity of the semigroup $S\left(\left[\frac{r}{s}, \frac{u}{v}\right]\right)$ to the common ancestor in the Stern-Brocot tree of $\frac{r}{s}$ and $\frac{u}{v}$ and we give an algorithm (also with similar complexity to the one of Euclides') to compute this ancestor and also the multiplicity of the corresponding proportionally modular numerical semigroup.

\section{BÉzout SEQuences}

We begin this section by establishing some notation. Given integers $a, b$ and $c$ we denote

$$
\left\lfloor\frac{a}{b}\right\rfloor=\max \left\{x \in \mathbb{Z} ; x \leq \frac{a}{b}\right\} \quad \text { and } \quad\left\lceil\frac{a}{b}\right\rceil=\min \left\{x \in \mathbb{Z} ; \frac{a}{b} \leq x\right\} .
$$

We will write $a \equiv b \bmod c$ if $a-b$ is a multiple of $c$ and $a=b \bmod c$ if $a<c$ and $a \equiv b \bmod c$. We also will write $a=b^{-1} \bmod c$ if $0<a<c$ and $a b \equiv 1 \bmod c$.

Let us note that given a Bézout sequence with decreasing numerators

$$
\frac{a}{b}<\frac{c}{d}
$$

$c b-a d=1$ and $a \geq c$, then

$$
\frac{d}{c}<\frac{b}{a}
$$

is a Bézout sequence with increasing denominators. In this sense we see sequence (4) as a dual of sequence (3). Every Bézout sequence with increasing denominators has a dual Bézout sequence with decreasing numerators (obtained by inverting every fraction and reversing their order). It follows that any result for a Bézout sequence with decreasing numerators has a corresponding dual, whose proof is obtained just by considering the corresponding dual sequence.

Lemma 2.1. A Bézout sequence with decreasing numerators has strictly decreasing denominators and a Bézout sequence with strictly increasing numerators has increasing denominators. Moreover, if

$$
\frac{a_{1}}{b_{1}}<\frac{a_{2}}{b_{2}}
$$

is a Bézout sequence with $a_{1}=a_{2}$, then $a_{1}=a_{2}=1$. 
Proof. Recall first that all numerators and denominators in Bézout sequences are positive integers. Given a sequence

$$
\frac{a_{1}}{b_{1}}<\frac{a_{2}}{b_{2}}
$$

with $a_{2} b_{1}-a_{1} b_{2}=1$.

- If $a_{1} \geq a_{2}$, then $\frac{a_{1}}{b_{1}}<\frac{a_{2}}{b_{2}}$ implies $b_{1}>b_{2}$.

- If $a_{1}<a_{2}$, then $1=a_{2} b_{1}-a_{1} b_{2}>a_{2} b_{1}-a_{2} b_{2}=a_{2}\left(b_{1}-b_{2}\right)$ and $2 \leq a_{2}$. Therefore $b_{1}-b_{2} \leq 0$, and so $b_{1} \leq b_{2}$. Observe that the denominators can be equal, as it happens with the sequence $\frac{a}{1}<\frac{a+1}{1}$.

- If $a_{1}=a_{2}$, then the equality $1=a_{2} b_{1}-a_{1} b_{2}$ implies $a_{1}=a_{2}=1$.

We have the following dual result.

Lemma 2.2 (Dual of Lemma 2.1). A Bézout sequence with increasing denominators has strictly increasing numerators and a Bézout sequence with strictly decreasing denominators has decreasing numerators. Moreover, if

$$
\frac{a_{1}}{b_{1}}<\frac{a_{2}}{b_{2}}
$$

is a Bézout sequence with $b_{1}=b_{2}$, then $b_{1}=b_{2}=1$.

The next proposition shows how to add an element to a Bézout sequence so that the resulting sequence is also a Bézout sequence.

Proposition 2.3. Given a Bézout sequence with decreasing numerators and right end $\frac{a}{b}$, if $b>1$, then there is a unique reduced fraction that can be joined to the right of the sequence in such a way that the resulting sequence is also a Bézout sequence with decreasing numerators. This fraction is $\frac{1}{b-1}$ if $a=1$, or $\frac{b^{-1} \bmod a}{(-a)^{-1} \bmod b}$ if $a>1$.

Proof. Given the sequence

$$
\frac{a_{1}}{b_{1}}<\frac{a_{2}}{b_{2}}
$$

the equality $a_{2} b_{1}-a_{1} b_{2}=1$ implies

$$
a_{2} b_{1} \equiv 1 \bmod a_{1} \quad \text { and } \quad-a_{1} b_{2} \equiv 1 \bmod b_{1} .
$$

Moreover:

- If $a_{1}=1$, then $a_{2}=1$ and $b_{2}=b_{1}-1$.

- If $a_{1}>1$ then, by Lemma 2.1, $a_{1}>a_{2}$ and so $a_{2} b_{1} \equiv 1 \bmod a_{1}$ implies $a_{2}=b_{1}^{-1} \bmod a_{1}$. Also by Lemma 2.1 $b_{1}>b_{2}$ and so $-a_{1} b_{2} \equiv 1 \bmod b_{1}$ implies $b_{2}=\left(-a_{1}\right)^{-1} \bmod b_{1}$.

Therefore, if we have a Bézout sequence with decreasing numerators and right end $\frac{a}{b}$ with $b>1$, to get a one term longer Bézout sequence with decreasing numerators, we can only join to the right of the sequence:

- The term $\frac{1}{b-1}$ whenever $a=1$, or

- The term $\frac{b^{-1} \bmod a}{(-a)^{-1} \bmod b}$ whenever $a>1$.

The following corollary is an immediate consequence of Proposition 2.3 . 
Corollary 2.4. A Bézout sequence with decreasing numerators is completely determined by its left end and its length. Therefore, there is at most one Bézout sequence with decreasing numerators connecting two fractions and, any Bézout sequence with decreasing numerators is proper.

The corresponding duals of Proposition 2.3 and Corollary 2.4 are the following.

Proposition 2.5 (Dual of Proposition 2.3). Given a Bézout sequence with increasing denominators and left end $\frac{c}{d}$, if $c>1$, then there is a unique fraction that can be joined to the left of the sequence in such a way that the resulting sequence is also a Bézout sequence with increasing denominators. This fraction is $\frac{c-1}{1}$ whenever $d=1$, or $\frac{(-d)^{-1} \bmod c}{c^{-1} \bmod d}$ whenever $d>1$.

Corollary 2.6 (Dual of Corollary 2.4). A Bézout sequence with increasing denominators is completely determined by its right end and its length. Therefore, there is at most one Bézout sequence with increasing denominators connecting two fractions, and any Bézout sequence with increasing denominators is proper.

We know from [6] that any two reduced fractions $\frac{a}{b}<\frac{c}{d}$ can be connected by a proper Bézout sequence and that any proper Bézout sequence, such as (2), is convex in the sense that there is an $h \in\{1, \ldots, k\}$ such that

$$
a_{1} \geq a_{2} \geq \cdots \geq a_{h} \leq a_{h+1} \leq \cdots \leq a_{k} .
$$

We may now refine the above as follows.

Theorem 2.7. Let $\frac{a}{b}<\frac{c}{d}$ be reduced fractions. Then, there is a unique proper Bézout sequence connecting them, which is obtained by concatenating a Bézout sequence with decreasing numerators and left end $\frac{a}{b}$ with a Bézout sequence with increasing denominators and right end $\frac{c}{d}$.

Proof. We know (see [6]) that there is a proper Bézout sequence connecting both fractions:

$$
\mathcal{B}=\left\{\frac{a}{b}=\frac{a_{1}}{b_{1}}<\frac{a_{2}}{b_{2}}<\cdots<\frac{a_{h}}{b_{h}}<\cdots<\frac{a_{k}}{b_{k}}=\frac{c}{d}\right\},
$$

which is convex, $a_{1} \geq a_{2} \geq \cdots \geq a_{h} \leq a_{h+1} \leq \cdots \leq a_{k}$. We can assume that $a_{h} \neq a_{h+1}$, otherwise $h$ is replaced by $h+1$. Then $a_{h+1} \neq 1$ and Lemma 2.1 yields $a_{h+1}<a_{h+2}$. Recursively, we have

$$
a_{h}<a_{h+1}<\cdots<a_{k} .
$$

By using again Lemma 2.1 we have $b_{h} \leq b_{h+1} \leq \cdots \leq b_{k}$ and therefore the sequence (5) is obtained by joining the Bézout sequence with decreasing numerators and left end $\frac{a}{b}$,

$$
\mathcal{B}_{\text {dec }}=\frac{a}{b}=\frac{a_{1}}{b_{1}}<\frac{a_{2}}{b_{2}}<\cdots<\frac{a_{h}}{b_{h}},
$$

to the Bézout sequence with increasing denominators and right end $\frac{c}{d}$,

$$
\mathcal{B}_{\text {inc }}=\frac{a_{h}}{b_{h}}<\cdots<\frac{a_{k}}{b_{k}}=\frac{c}{d} .
$$

If we prove that $h$ and $k-h$ only depend on $\frac{a}{b}$ and $\frac{c}{d}$, then Corollaries 2.4 and 2.6 complete the proof of this theorem. 
Let us suppose that we can find another proper Bézout sequence

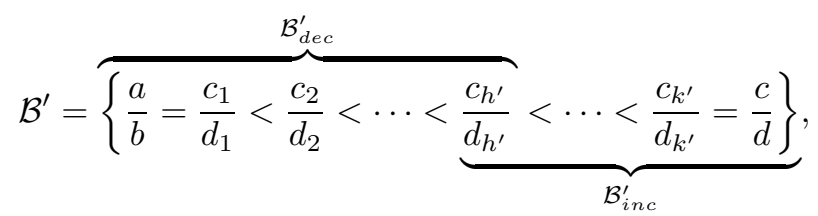

with $c_{1} \geq c_{2} \geq \cdots \geq c_{h^{\prime}}<c_{h^{\prime}+1} \cdots<c_{k^{\prime}}$ which also connects $\frac{a}{b}$ and $\frac{c}{d}$.

We are going to prove that neither of the following cases can be true, and then the unique remaining case is $h=h^{\prime}$ and $k-h=k^{\prime}-h^{\prime}$.

(i) If $h<h^{\prime}$ and $k-h \leq k^{\prime}-h^{\prime}$, then Corollaries 2.4 and 2.6 imply that $\mathcal{B}_{\text {dec }} \varsubsetneqq$ $\mathcal{B}_{\text {dec }}^{\prime}$ and $\mathcal{B}_{\text {inc }} \subseteq \mathcal{B}_{\text {inc }}^{\prime}$. Therefore, the sequence (5) is a Bezóut subsequence of (6) which contradicts that (6) is proper. A similar contradiction arises when $h \leq h^{\prime}$ and $k-h<k^{\prime}-h^{\prime}$.

(ii) If $h<h^{\prime}$ and $k^{\prime}-h^{\prime} \leq k-h$, then in view of Corollaries 2.4 and 2.6 we have that $\mathcal{B}_{\text {dec }} \varsubsetneqq \mathcal{B}_{\text {dec }}^{\prime}$ (as a left tail) and $\mathcal{B}_{\text {inc }}^{\prime} \subseteq \mathcal{B}_{\text {inc }}$ (as a right tail). Therefore, $\frac{a_{h}}{b_{h}}=\frac{c_{h}}{d_{h}}$ and $\frac{c_{h^{\prime}}}{d_{h^{\prime}}}=\frac{a_{k-k^{\prime}+h^{\prime}}}{b_{k-k^{\prime}+h^{\prime}}}$ and we have

$$
c_{h} \geq c_{h+1} \geq \cdots \geq c_{h^{\prime}}
$$

and

$$
c_{h}=a_{h}<a_{h+1}<\cdots<a_{k-k^{\prime}+h^{\prime}}=c_{h^{\prime}},
$$

which is clearly a contradiction. The same holds for $h \leq h^{\prime}$ and $k^{\prime}-h^{\prime}<$ $k-h$.

(iii) The proofs for the cases $h^{\prime}<h$ and $k^{\prime}-h^{\prime} \leq k-h$, or $h^{\prime} \leq h$ and $k^{\prime}-h^{\prime}<k-h$ are as in (i) but interchanging sequences (6) and (5).

(iv) The proofs for the cases $h^{\prime}<h$ and $k-h \leq k^{\prime}-h^{\prime}$, or $h^{\prime} \leq h$ and $k-h<k^{\prime}-h^{\prime}$ follow as in (ii) but interchanging sequences (6) and (5).

We conclude this section by noting that the element $a_{h}$ found in Theorem 2.7 above is an invariant of the semigroup $S\left(\left[\frac{a}{b}, \frac{c}{d}\right]\right)$, in fact, $a_{h}$ is the multiplicity of the semigroup. Also, two other invariants of the above semigroup were also found, namely $h$ and $k$.

\section{An Algorithm to COMPute the unique Proper BÉzout SEQuence CONNECTING TWO REDUCED FRACTIONS}

As we have proved in Theorem 2.7 the proper Bézout sequence connecting two reduced fractions is obtained by merging a Bézout sequence with decreasing numerators with a Bézout sequence with increasing denominators. Thus, Proposition 2.3 (Proposition 2.5) shows that a Bézout sequence with decreasing numerators (increasing denominators) is completely determined by its left (right) end and its length. These propositions also give us a recursive method to compute the terms of such Bézout sequences. We begin this section by computing the longest Bézout sequence with decreasing numerators (increasing denominators) and fixed left (right) end, but first we give a couple of lemmas that simplify the computation of the terms in such sequences. 
Lemma 3.1. Given a Bézout sequence with decreasing numerators

$$
\frac{a_{1}}{b_{1}}<\frac{a_{2}}{b_{2}}<\frac{a_{3}}{b_{3}}
$$

then $b_{3}=-b_{1} \bmod b_{2}$. Moreover, if $a_{2} \neq 1$, then $a_{3}=-a_{1} \bmod a_{2}$.

Proof. The Bézout condition asserts that $a_{2} b_{1}-a_{1} b_{2}=1=a_{3} b_{2}-a_{2} b_{3}$. Then, by taking $\bmod b_{2}$ and $\bmod a_{2}$, we have

$$
a_{2} b_{1} \equiv-a_{2} b_{3} \bmod b_{2} \quad \text { and } \quad-a_{1} b_{2} \equiv a_{3} b_{2} \bmod a_{2},
$$

respectively. Moreover, $a_{2}$ is a unit module $b_{2}$ and $b_{2}$ is a unit module $a_{2}$, so we also have

$$
b_{3} \equiv-b_{1} \bmod b_{2} \quad \text { and } \quad a_{3} \equiv-a_{1} \bmod a_{2} .
$$

Lemma 2.1 ensures that $b_{1}>b_{2}>b_{3}$, and thus $b_{3}=-b_{1} \bmod b_{2}$. Moreover, if $a_{2} \neq 1$, then Lemma 2.1 also implies $a_{1}>a_{2}>a_{3}$. Hence $a_{3}=-a_{1} \bmod a_{2}$.

Dually we obtain the following.

Lemma 3.2 (Dual of Lemma 3.1). Given a Bézout sequence with increasing denominators

$$
\frac{a_{1}}{b_{1}}<\frac{a_{2}}{b_{2}}<\frac{a_{3}}{b_{3}}
$$

then $a_{1}=-a_{3} \bmod a_{2}$. Moreover, if $b_{2} \neq 1$, then $b_{1}=-b_{3} \bmod b_{2}$.

The next proposition gives the longest Bézout sequence with decreasing numerators and a fixed left end.

Proposition 3.3. Let $\mathcal{B}$ be the longest Bézout sequence with decreasing numerators and left end $\frac{a}{b}$.

(i) If $a=1$, then the sequence $\mathcal{B}$ is

$$
\frac{1}{b}<\frac{1}{b-1}<\frac{1}{b-2}<\cdots<\frac{1}{1} .
$$

(ii) If $b=1$, then the sequence $\mathcal{B}$ only has the term, $\frac{a}{1}$.

(iii) If $a>b$, then the sequence $\mathcal{B}$ has strictly decreasing numerators, the ceiling function, $\lceil-\rceil$, is constant on $\mathcal{B}$ and its right end is $\frac{\left\lceil\frac{a}{b}\right\rceil}{1}$.

(iv) If $a<b$, then the sequence $\mathcal{B}$ has right end $\frac{1}{1}$, it has $\frac{1}{\left[\frac{b}{a}\right]}$ as a term and the floor function, $\lfloor-\rfloor$, is constant on the inverses of the fractions in the subsequence of $\mathcal{B}$ with right end $\frac{1}{\left\lfloor\frac{b}{a}\right\rfloor}$.

Proof. The cases $a=1$ or $b=1$ are clear.

Case 1) If $a>b$. Then $1<\frac{a}{b}$ and so, for any fraction $\frac{a_{i}}{b_{i}}$ in $\mathcal{B}$, we also have $1<\frac{a_{i}}{b_{i}}$.

Given two consecutive fractions in $\mathcal{B}$,

$$
\frac{a_{i}}{b_{i}}<\frac{a_{i+1}}{b_{i+1}}
$$

if $a_{i}=a_{i+1}$, then the equality $a_{i+1} b_{i}-a_{i} b_{i+1}=1$ implies $a_{i}=a_{i+1}=1$, and this contradicts $1<\frac{a_{i}}{b_{i}}$. Therefore, the sequence $\mathcal{B}$ has strictly decreasing numerators. 
Thus, if $b_{i+1}=1$, then we have $\frac{a_{i}}{b_{i}}=a_{i+1}-\frac{1}{b_{i}}$, with $b_{i} \geq 2$, and so $\left\lceil\frac{a_{i}}{b_{i}}\right\rceil=$ $a_{i+1}=\left[\frac{a_{i+1}}{b_{i+1}}\right]$; if $b_{i+1} \geq 2$, then we have $\frac{a_{i+1}}{b_{i+1}}=\frac{a_{i}}{b_{i}}+\frac{1}{b_{i} b_{i+1}}$, which also leads to $\left\lceil\frac{a_{i}}{b_{i}}\right\rceil=\left\lceil\frac{a_{i+1}}{b_{i+1}}\right\rceil$.

To conclude (iii) we use Proposition 2.3 and Lemma 3.1 to add recursively new terms to the right of $\frac{a}{b}$ until we get a Bézout sequence with decreasing numerators whose right end has 1 as denominator. Note that the ceiling is constant and equal to $\left\lceil\frac{a}{b}\right\rceil$ on the terms we are joining and so the last term we can append to the right of the sequence is $\frac{\left\lceil\frac{a}{b}\right\rceil}{1}$.

Case 2) If $a<b$. Given two consecutive fractions in $\mathcal{B}$, as above, if $a_{i}>1$ and $a_{i+1}=1$, then we have $b_{i}-a_{i} b_{i+1}=1$. So $\frac{b_{i}}{a_{i}}=b_{i+1}+\frac{1}{a_{i}}$ and $\left\lfloor\frac{b_{i}}{a_{i}}\right\rfloor=b_{i+1}=\left\lfloor\frac{b_{i+1}}{a_{i+1}}\right\rfloor$.

If $a_{i+1}>1$, then $a_{i}>a_{i+1}$, and $\frac{b_{i+1}}{a_{i+1}}=\frac{b_{i}}{a_{i}}-\frac{1}{a_{i} a_{i+1}}$ implies $\left\lfloor\frac{b_{i}}{a_{i}}\right\rfloor=\left\lfloor\frac{b_{i+1}}{a_{i+1}}\right\rfloor$.

To conclude (iv) we use Proposition 2.3 and Lemma 3.1 to add recursively new terms $\frac{c}{d}$, with $\left\lfloor\frac{d}{c}\right\rfloor=\left\lfloor\frac{b}{a}\right\rfloor$, to the right of $\frac{a}{b}$ until we get a Bézout sequence with decreasing numerators whose right end has numerator 1 (and denominator $\left\lfloor\frac{b}{a}\right\rfloor$ ). Then we append terms, to the right, with numerators 1 and decreasing denominators, until we reach $\frac{1}{1}$. The sequence $\mathcal{B}$ is

$$
\frac{a}{b}<\cdots<\frac{1}{\left\lfloor\frac{b}{a}\right\rfloor}<\frac{1}{\left\lfloor\frac{b}{a}\right\rfloor-1}<\cdots<\frac{1}{1} .
$$

To produce the longest Bézout sequence with decreasing numerators and left end the reduced fraction $\frac{a_{1}}{b_{1}}\left(a_{1} \neq 1\right.$ and $\left.b_{1} \neq 1\right)$, we proceed as follows.

Case 1) $a_{1}>b_{1}$. First we calculate $a_{2}=b_{1}^{-1} \bmod a_{1}$ and $b_{2}=\left(-a_{1}\right)^{-1} \bmod b_{1}$, and recursively we take $a_{i+2}=-a_{i} \bmod a_{i+1}$ and $b_{i+2}=-b_{i} \bmod b_{i+1}$ until we reach $\left\lceil\frac{a_{1}}{b_{1}}\right]$ with the $a$ 's (and 1 with the $b$ 's).

For example, to get the longest Bézout sequence with decreasing numerators and right end $\frac{131}{50}$ we calculate

\begin{tabular}{|l|l|}
\hline$a_{i}$ & $b_{i}$ \\
\hline 131 & 50 \\
$76=50^{-1} \bmod 131$ & $29=(-131)^{-1} \bmod 50$ \\
$21=-131 \bmod 76$ & $8=-50 \bmod 29$ \\
$8=-76 \bmod 21$ & $3=-29 \bmod 8$ \\
$3=-21 \bmod 8=\left[\frac{131}{50}\right]$ & $1=-8 \bmod 3$ \\
\hline
\end{tabular}

So the sequence is

$$
\frac{131}{50}<\frac{76}{29}<\frac{21}{8}<\frac{8}{3}<\frac{3}{1}
$$

Case 2) $a_{1}<b_{1}$. As in the above case we first calculate $a_{2}=b_{1}^{-1} \bmod a_{1}$ and $b_{2}=\left(-a_{1}\right)^{-1} \bmod b_{1}$, and recursively we take $a_{i+2}=-a_{i} \bmod a_{i+1}$ and $b_{i+2}=$ $-b_{i} \bmod b_{i+1}$ until we reach $\left\lfloor\frac{b_{1}}{a_{1}}\right\rfloor$ with the $b$ 's (and 1 with the $a$ 's). Finally, we add 
terms to the sequence with 1 as numerators and strictly decreasing denominators until we reach $\frac{1}{1}$.

For example, to get the longest Bézout sequence with decreasing numerators and left end $\frac{34}{111}$ we calculate

\begin{tabular}{|l|l|}
\hline$a_{i}$ & $b_{i}$ \\
\hline 34 & 111 \\
$19=111^{-1} \bmod 34$ & $62=(-34)^{-1} \bmod 111$ \\
$4=-34 \bmod 19$ & $13=-111 \bmod 62$ \\
$1=-19 \bmod 4$ & $3=-62 \bmod 13=\left\lfloor\frac{111}{34}\right\rfloor$ \\
1 & $2=3-1$ \\
1 & $1=2-1$ \\
\hline
\end{tabular}

So the sequence is

$$
\frac{34}{111}<\frac{19}{62}<\frac{4}{13}<\frac{1}{3}<\frac{1}{2}<\frac{1}{1}
$$

In the same way, the dual of Proposition 3.3 gives the longest Bézout sequence with increasing denominators and right end $\frac{c}{d}$.

Proposition 3.4 (Dual of Proposition 3.3). Let $\mathcal{B}^{\prime}$ be the longest Bézout sequence with increasing denominators and right end $\frac{c}{d}$. Then:

(i) If $d=1$, the sequence $\mathcal{B}^{\prime}$ is

$$
\frac{1}{1}<\cdots<\frac{c-2}{1}<\frac{c-1}{1}<\frac{c}{1}
$$

(ii) If $c=1$, the sequence $\mathcal{B}^{\prime}$ only has the term $\frac{1}{d}$.

(iii) If $c<d$, the sequence $\mathcal{B}^{\prime}$ has strictly increasing denominators, the function $\lceil-\rceil$ is constant on the inverses of the fractions in $\mathcal{B}^{\prime}$ and its left end is $\frac{1}{\left\lceil\frac{d}{c}\right\rceil}$.

(iv) If $c>d$, the sequence $\mathcal{B}^{\prime}$ has left end $\frac{1}{1}$, it has $\frac{\left\lfloor\frac{c}{d}\right\rfloor}{1}$ as a term and the function $\lfloor-\rfloor$ is constant on the fractions in the subsequence of $\mathcal{B}^{\prime}$ with left end $\frac{\left\lfloor\frac{c}{d}\right\rfloor}{1}$.

Then to produce the longest Bézout sequence with increasing denominators and right end the reduced fraction $\frac{c_{1}}{d_{1}}\left(c_{1} \neq 1\right.$ and $\left.d_{1} \neq 1\right)$ we proceed as follows.

Case 1) $c_{1}<d_{1}$. First we calculate $d_{2}=c_{1}^{-1} \bmod d_{1}$ and $c_{2}=\left(-d_{1}\right)^{-1} \bmod c_{1}$, and recursively we take $d_{i+2}=-d_{i} \bmod d_{i+1}$ and $c_{i+2}=-c_{i} \bmod c_{i+1}$ until we reach $\left\lceil\frac{d_{1}}{c_{1}}\right\rceil$ with the $d$ 's (and 1 with the $c$ 's). 
For example, to get the longest Bézout sequence with increasing denominators and right end $\frac{35}{46}$ we calculate

\begin{tabular}{|l|l|}
\hline$c_{i}$ & $d_{i}$ \\
\hline 35 & 46 \\
$19=(-46)^{-1} \bmod 35$ & $25=35^{-1} \bmod 46$ \\
$3=-35 \bmod 19$ & $4=-46 \bmod 25$ \\
$2=-19 \bmod 3$ & $3=-25 \bmod 4$ \\
$1=-3 \bmod 2$ & $2=-4 \bmod 3=\left\lceil\frac{46}{35}\right\rceil$ \\
\hline
\end{tabular}

So the sequence is

$$
\frac{1}{2}<\frac{2}{3}<\frac{3}{4}<\frac{19}{25}<\frac{35}{46}
$$

Case 2) $c_{1}>d_{1}$. As in the above case we first calculate $d_{2}=c_{1}^{-1} \bmod d_{1}$ and $c_{2}=\left(-d_{1}\right)^{-1} \bmod c_{1}$, and recursively we take $d_{i+2}=-d_{i} \bmod d_{i+1}$ and $c_{i+2}=$ $-c_{i} \bmod c_{i+1}$ until we reach $\left\lfloor\frac{c_{1}}{d_{1}}\right\rfloor$ with the $c^{\prime}$ 's (and 1 with the $d$ 's). Finally, we join terms with 1 as denominators and strictly decreasing numerators until we reach $\frac{1}{1}$.

For example, to get the longest Bézout sequence with increasing denominators and right end $\frac{131}{47}$, we calculate

\begin{tabular}{|l|l|}
\hline$c_{i}$ & $d_{i}$ \\
\hline 131 & 47 \\
$39=(-47)^{-1} \bmod 131$ & $14=(-34)^{-1} \bmod 111$ \\
$25=-131 \bmod 39$ & $9=-47 \bmod 14$ \\
$11=-39 \bmod 25$ & $4=-14 \bmod 9$ \\
$8=-25 \bmod 11$ & $3=-9 \bmod 4$ \\
$5=-11 \bmod 8$ & $2=-4 \bmod 3$ \\
$2=-8 \bmod 5=\left\lfloor\frac{131}{47}\right\rfloor$ & 1 \\
$1=2-1$ & 1 \\
\hline
\end{tabular}

So the sequence is

$$
\frac{1}{1}<\frac{2}{1}<\frac{5}{2}<\frac{8}{3}<\frac{11}{4}<\frac{25}{9}<\frac{39}{14}<\frac{131}{47} .
$$

We summarize the above results in the following algorithm.

\section{Algorithm 3.5.}

INPUT: Two reduced fractions $\frac{a}{b}<\frac{c}{d}$.

Output: The unique proper Bézout sequence with left and right ends $\frac{a}{b}$ and $\frac{c}{d}$ respectively.

(1) Compute $\mathcal{B}_{\text {ldec }}$, the longest Bézout sequence with decreasing numerators and left end $\frac{a}{b}$. 
(2) Let $\mathcal{B}_{\text {inc }}=\left\{\frac{c}{d}\right\}$ and $\frac{m}{n}$ the left end of $\mathcal{B}_{\text {inc }}$.

(3) While $\frac{m}{n} \notin \mathcal{B}_{\text {ldec }}$ compute the unique element $\frac{x}{y}$ that can be joined to the left of $\mathcal{B}_{\text {inc }}$ to get a one term longer Bézout sequence with increasing denominators, and replace $\mathcal{B}_{\text {inc }}$ with $\left\{\frac{x}{y}\right\} \cup \mathcal{B}_{\text {inc }}$.

(4) Let $\mathcal{B}_{\text {dec }}$ be the subsequence of $\mathcal{B}_{\text {ldec }}$ with left and right ends $\frac{a}{b}$ and $\frac{m}{n}$, respectively.

(5) Return the concatenation of $\mathcal{B}_{\text {dec }}$ and $\mathcal{B}_{\text {inc }}$.

\section{The Stern-Brocot tree and Bézout sequences}

The Stern-Brocot tree provides a recursive method to list all reduced fractions $\frac{x}{y}$ with $x$ and $y$ positive integers [2].

To build the Stern-Brocot tree we start with the expressions

$$
\frac{0}{1}, \frac{1}{0}
$$

representing 0 and infinity. Between two adjacent expressions $\frac{m}{n}$ and $\frac{m^{\prime}}{n^{\prime}}$, we insert its mediant $\frac{m+m^{\prime}}{n+n^{\prime}}$. We recursively obtain sequences

$$
\begin{gathered}
\frac{0}{1}<\frac{1}{1}<\frac{1}{0} \\
\frac{0}{1}<\frac{1}{2}<\frac{1}{1}<\frac{2}{1}<\frac{1}{0}, \\
\frac{0}{1}<\frac{1}{3}<\frac{1}{2}<\frac{2}{3}<\frac{1}{1}<\frac{3}{2}<\frac{2}{1}<\frac{3}{1}<\frac{1}{0},
\end{gathered}
$$

which are all Bézout sequences (see [2]). Finally, we connect a mediant with the two fractions used to compute it:

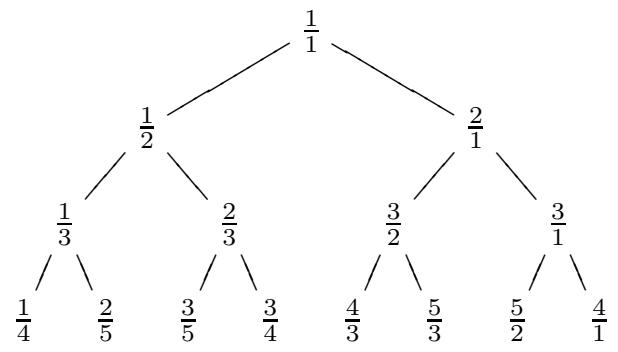

Any reduced fraction appears just once in the Stern-Brocot tree. There is an easy algorithm to obtain the path between $1 / 1$ and any other fraction $m / n$ (see [1]).

\section{Algorithm 4.1.}

$$
\begin{aligned}
& \text { While } m \neq n \text { do } \\
& \text { If } m<n \\
& \quad \text { then (output }(L) ; \text { replace } n \text { with } n-m) \\
& \quad \text { else (output }(R) \text {; replace } m \text { with } m-n)
\end{aligned}
$$

For example, the path between $1 / 1$ and $11 / 5$ is $R R L L L L$, which means: from $1 / 1$ take right to $2 / 1$ then right again to $3 / 1$, then left to $5 / 2$ and so on.

To see where a path $W$ leads, we only have to replace $L$ and $R$ in $W$ by the matrices

$$
\mathbf{L}=\left(\begin{array}{ll}
1 & 1 \\
0 & 1
\end{array}\right) \quad \text { and } \quad \mathbf{R}=\left(\begin{array}{ll}
1 & 0 \\
1 & 1
\end{array}\right)
$$


and so by multiplying we obtain a new matrix $\mathbf{W}$. Then the path $W$ leads to the fraction represented by

$$
\left(\begin{array}{ll}
0 & 1 \\
1 & 0
\end{array}\right) \mathbf{W}\left(\begin{array}{l}
1 \\
1
\end{array}\right)
$$

(see also [1]).

For example, the path $W=R R L L L L$ leads to the fraction represented by the product

$$
\left(\begin{array}{ll}
0 & 1 \\
1 & 0
\end{array}\right) \operatorname{RRLLLL}\left(\begin{array}{l}
1 \\
1
\end{array}\right)=\left(\begin{array}{c}
11 \\
5
\end{array}\right)
$$

that is, $\frac{11}{5}$.

We now point out two properties of the leaves in the Stern-Brocot tree.

- If $\frac{m_{L}}{n_{L}}$ and $\frac{m_{R}}{n_{R}}$ are respectively the left and right sons of $\frac{m}{n}$,

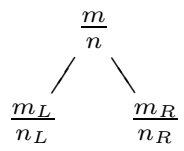

then,

$$
\frac{m_{L}}{n_{L}}<\frac{m}{n}<\frac{m_{R}}{n_{R}}
$$

is a Bézout sequence. Hence

$$
\frac{m_{L}}{n_{L}}<\frac{m}{n}
$$

is a Bézout sequence with decreasing numerators, and therefore with strictly decreasing denominators. Analogously,

$$
\frac{m}{n}<\frac{m_{R}}{n_{R}}
$$

is a Bézout sequence with increasing denominators, and thus with strictly increasing numerators.

- In the situation

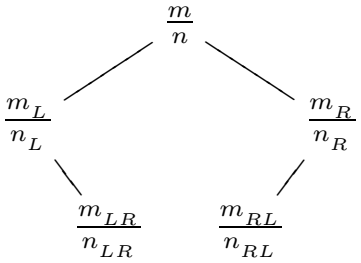

we have a Bézout sequence

$$
\frac{m_{L}}{n_{L}}<\frac{m_{L R}}{n_{L R}}=\frac{m_{L}+m}{n_{L}+n}<\frac{m}{n}<\frac{m_{R L}}{n_{R L}}=\frac{m_{R}+m}{n_{R}+n}<\frac{m_{R}}{n_{R}},
$$

and therefore

$$
\frac{m_{L R}}{n_{L R}}<\frac{m}{n}
$$

is a Bézout sequence with decreasing numerators, and

$$
\frac{m}{n}<\frac{m_{R L}}{n_{R L}}
$$

is a Bézout sequence with increasing denominators. 
The next proposition extends the above two properties. Its proof is constructive and it will furnish the basis for an algorithmic method to connect two reduced fractions by a proper Bézout sequence.

Proposition 4.2. Given reduced fractions $\frac{r}{s}<\frac{u}{v}$ :

(1) The fraction $\frac{r}{s}$ is a descendent of $\frac{u}{v}$ in the Stern-Brocot tree if and only if $\frac{r}{s}$ and $\frac{u}{v}$ are connected by a Bézout sequence with decreasing numerators.

(2) The fraction $\frac{u}{v}$ is a descendent of $\frac{r}{s}$ in the Stern-Brocot tree if and only if $\frac{r}{s}$ and $\frac{u}{v}$ are connected by a Bézout sequence with increasing denominators.

Proof. Note that if we consider the path

$$
\left(\frac{m}{n}, \frac{m_{L}}{n_{L}}, \frac{m_{L R}}{n_{L R}}, \ldots, \frac{m_{L R . i . R}}{n_{L R . i . R}}\right)
$$

in the Stern-Brocot tree, with $1 \leq i$, we have

$$
\frac{m_{L}}{n_{L}}<\frac{m_{L R}}{n_{L R}}<\ldots<\frac{m_{L R . i . R}}{n_{L R . i . R}}<\frac{m}{n}
$$

with

for all $1 \leq j \leq i$. Hence, by induction,

$$
\frac{m_{L R, R}^{j}}{n_{L R, R_{R}^{j}}}=\frac{m_{L R^{j-1} R}+m}{n_{L R^{j-1} R}+n},
$$

$$
\frac{m_{L R{ }^{j} R}}{n_{L R{ }^{j} \cdot R}}<\frac{m}{n}
$$

is a Bézout sequence with decreasing numerators. Dually, given the path

$$
\left(\frac{m}{n}, \frac{m_{R}}{n_{R}}, \frac{m_{R L}}{n_{R L}}, \ldots, \frac{m_{R L . i . L}}{n_{R L . i . L}}\right),
$$

with $1 \leq i$,

$$
\frac{m}{n}<\frac{m_{R L . j . L}}{n_{R L . j . L}}
$$

is a Bézout sequence with increasing denominators, for all $1 \leq j \leq i$.

(1) Let us suppose that $\frac{r}{s}$ is a descendent of $\frac{u}{v}$ and let

$$
P=\left(\frac{u}{v}=\frac{c_{1}}{d_{1}}, \frac{c_{2}}{d_{2}}, \ldots, \frac{c_{k}}{d_{k}}=\frac{r}{s}\right)
$$

be the descending path in the Stern-Brocot tree connecting both fractions. Then $c_{2}=u_{L}$ and $d_{2}=v_{L}$. To get a Bézout sequence with decreasing numerators connecting both fractions, we remove from $P$ the fraction $\frac{c_{i}}{d_{i}}$ whenever the fraction $\frac{c_{i+1}}{d_{i+1}}$ is on its right, $1<i \leq k-1$. The resulting sequence (sorted in descending order) is a Bézout sequence with decreasing numerators connecting $\frac{r}{s}$ and $\frac{u}{v}$.

(2) Dually, if $\frac{u}{v}$ is a descendent of $\frac{r}{s}$, and

$$
P^{\prime}=\left(\frac{r}{s}=\frac{a_{1}}{b_{1}}, \frac{a_{2}}{b_{2}}, \ldots, \frac{a_{k}}{b_{k}}=\frac{u}{v}\right)
$$

is the descending path in the Stern-Brocot tree connecting both fractions, then $a_{2}=r_{R}$ and $b_{2}=s_{R}$. To get the Bézout sequence with increasing 
denominators connecting both fractions, we remove from $P^{\prime}$ the fraction $\frac{a_{i}}{b_{i}}$ whenever the fraction $\frac{a_{i+1}}{b_{i+1}}$ is on its left, $1<i \leq k-1$. The resulting sequence is the Bézout sequence with increasing denominators connecting $\frac{r}{s}$ and $\frac{u}{v}$.

Conversely, since any two reduced fractions $\frac{r}{s}$ and $\frac{u}{v}$ appear just once in the Stern-Brocot tree, there is a unique path connecting both fractions. If we call $\frac{m}{n}$ to the common ancestor of $\frac{r}{s}$ and $\frac{u}{v}$, then

$$
\frac{r}{s}<\frac{m}{n}<\frac{u}{v}
$$

Thus (in view of the first part of this proof) $\frac{r}{s}$ and $\frac{m}{n}$ are connected by a Bézout sequence with decreasing numerators, and $\frac{m}{n}$ and $\frac{u}{v}$ are connected by a Bézout sequence with increasing denominators (and so, by Proposition 2.5, strictly increasing numerators). Since any two elements are connected by a unique proper Bézout sequence (Theorem 2.7) the unique way of connecting $\frac{r}{s}$ and $\frac{u}{v}$ by a Bézout sequence with decreasing numerators or a Bézout sequence with increasing denominators is that $\frac{m}{n}=\frac{u}{v}$ or $\frac{m}{n}=\frac{r}{s}$, respectively, and so $\frac{r}{s}$ is a descendent of $\frac{u}{v}$ or vice versa.

We can use the Stern-Brocot tree to get the proper Bézout sequence connecting two reduced fractions $\frac{r}{s}<\frac{u}{v}$ as follows:

- First apply Algorithm 4.1 to obtain the paths $P_{1}$ and $P_{2}$ connecting $\frac{1}{1}$ to $\frac{r}{s}$ and $\frac{u}{v}$, respectively.

- Then take $P$ as the beginning common part of $P_{1}$ and $P_{2}$.

- The right end $\frac{m}{n}$ of the path $P$ is the common ancestor of $\frac{r}{s}$ and $\frac{u}{v}$.

- The paths $P_{1} \backslash P$ and $P_{2} \backslash P$ (obtained by deleting $P$ in $P_{1}$ and $P_{2}$, respectively) connect $\frac{m}{n}$ to $\frac{r}{s}$ and $\frac{u}{v}$, respectively.

- Finally, apply the method in Proposition 4.2 to $P_{1} \backslash P$ and $P_{2} \backslash P$ to obtain the Bézout sequence with decreasing numerators and the Bézout sequence with increasing denominators connecting $\frac{m}{n}$ to $\frac{r}{s}$ and $\frac{u}{v}$, respectively, and join both sequences.

If we apply the above procedure to $\frac{33}{25}$ and $\frac{27}{7}$, we have:

- $P_{1}=R L L L R R R R R R R, P_{2}=R R R L R R R R R$.

- $P=R$.

- The common ancestor is the right end of $P$, that is, $\frac{2}{1}$.

- The sequences $P_{1} \backslash P=L L L R R R R R R R$ and $P_{2} \backslash P=R R L R R R R R$ connect $\frac{2}{1}$ to $\frac{33}{25}$ and $\frac{27}{7}$, respectively.

- If we apply the method in Proposition 4.2 to the paths $P_{1} \backslash P$ and $P_{2} \backslash P$, we obtain the sequences

$$
\frac{33}{25} \stackrel{L R R R R R R R}{\longleftarrow} \frac{4}{3} \stackrel{L}{\longleftarrow} \frac{3}{2} \stackrel{L}{\longleftarrow} \frac{2}{1}
$$

and

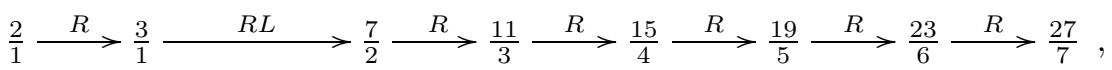

and the Bézout sequence connecting $\frac{33}{25}$ to $\frac{27}{7}$ is

$$
\frac{33}{25}<\frac{4}{3}<\frac{3}{2}<\frac{2}{1}<\frac{3}{1}<\frac{7}{2}<\frac{11}{3}<\frac{15}{4}<\frac{19}{5}<\frac{23}{6}<\frac{27}{7} .
$$




\section{The MUltiplicity of A PROPORTIONALly MODUlAR NUMERICAL SEMigroup}

In this section we apply the study we have done in the previous sections to give two algorithms. The first one computes the common ancestor in the Stern-Brocot tree of two reduced fractions and the second one computes the multiplicity of a proportionally modular numerical semigroup.

Algorithm 5.1 (To compute the common ancestor).

INPUT: Two reduced fractions $\frac{a}{b}$ and $\frac{c}{d}$.

Output: The common ancestor in the Stern-Brocot tree of $\frac{a}{b}$ and $\frac{c}{d}$.

(1) $\left(a_{1}, b_{1}\right)=(a, b), \quad\left(c_{1}, d_{1}\right)=(c, d), \quad M=\left(\begin{array}{ll}0 & 1 \\ 1 & 0\end{array}\right)$.

(2) If $b_{1}<a_{1}$ and $d_{1}<c_{1}$, then

$$
\left(a_{1}, b_{1}\right):=\left(a_{1}-b_{1}, b_{1}\right), \quad\left(c_{1}, d_{1}\right):=\left(c_{1}-d_{1}, d_{1}\right), \quad M:=M\left(\begin{array}{ll}
1 & 0 \\
1 & 1
\end{array}\right)
$$

and goto 2.

(3) If $a_{1}<b_{1}$ and $c_{1}<d_{1}$, then

$$
\left(a_{1}, b_{1}\right):=\left(a_{1}, b_{1}-a_{1}\right), \quad\left(c_{1}, d_{1}\right):=\left(c_{1}, d_{1}-a_{1}\right), \quad M:=M\left(\begin{array}{ll}
1 & 1 \\
0 & 1
\end{array}\right)
$$

and goto 2.

(4) Return mediant $(M)$, where

$$
\text { mediant }\left(\begin{array}{ll}
m_{11} & m_{12} \\
m_{21} & m_{22}
\end{array}\right)=\frac{m_{11}+m_{12}}{m_{21}+m_{22}} .
$$

Proposition 5.2. If $\frac{m}{n}$ is the common ancestor of the reduced fractions $\frac{a}{b}<\frac{c}{d}$ in the Stern-Brocot tree, then $m$ is the multiplicity of the proportionally modular numerical semigroup $S\left(\left[\frac{a}{b}, \frac{c}{d}\right]\right)$.

Proof. Note only that by Proposition 4.2 the common ancestor of $\frac{a}{b}$ and $\frac{c}{d}$ in the Stern-Brocot tree is a term of the longest Bézout sequence with decreasing numerators and left end $\frac{a}{b}$, and also a term of the longest Bézout sequence with increasing denominators and right end $\frac{c}{d}$. Then by Theorem 2.7 the unique Bézout sequence connecting $\frac{a}{b}$ and $\frac{c}{d}$ has the form

$$
\frac{a}{b}=\frac{a_{1}}{b_{1}}<\frac{a_{2}}{b_{2}}<\cdots<\frac{a_{h}}{b_{h}}=\frac{m}{n}<\cdots<\frac{a_{k}}{b_{k}}=\frac{c}{d}
$$

with

$$
a=a_{1} \geq a_{2} \geq \cdots \geq a_{h}=m<a_{h+1}<\cdots a_{k}=c .
$$

Furthermore, $S\left(\left[\frac{a}{b}, \frac{c}{d}\right]\right)=\left\langle a_{1}, a_{2}, \ldots, a_{h}, \ldots, a_{k}\right\rangle$ and then $m$ is the smallest positive integer that is in the semigroup $S\left(\left[\frac{a}{b}, \frac{c}{d}\right]\right)$ which is, by definition, the multiplicity of the semigroup.

Algorithm 5.3 (To compute the multiplicity).

INPUT: Two reduced fractions $\frac{a}{b}<\frac{c}{d}$.

OutPut: The multiplicity of the proportionally modular numerical semigroup $S\left(\left[\frac{a}{b}, \frac{c}{d}\right]\right)$.

(1) $\left(a_{1}, b_{1}\right)=(a, b), \quad\left(c_{1}, d_{1}\right)=(c, d), \quad\left(m_{1}, m_{2}\right)=(0,1)$. 
(2) If $b_{1}<a_{1}$ and $d_{1}<c_{1}$, then

$\left(a_{1}, b_{1}\right):=\left(a_{1}-b_{1}, b_{1}\right), \quad\left(c_{1}, d_{1}\right):=\left(c_{1}-d_{1}, d_{1}\right), \quad\left(m_{1}, m_{2}\right):=\left(m_{1}+m_{2}, m_{2}\right)$ and goto 2 .

(3) If $a_{1}<b_{1}$ and $c_{1}<d_{1}$, then

$\left(a_{1}, b_{1}\right):=\left(a_{1}, b_{1}-a_{1}\right), \quad\left(c_{1}, d_{1}\right):=\left(c_{1}, d_{1}-c_{1}\right), \quad\left(m_{1}, m_{2}\right):=\left(m_{1}, m_{1}+m_{2}\right)$

and goto 2.

(4) Return $m_{1}+m_{2}$.

For example, to compute the smallest positive integer that is solution the of the inequality $50 x \bmod 131 \leq 3 x$, we compute the multiplicity of the proportionally modular numerical semigroup $S\left(\left[\frac{131}{50}, \frac{131}{47}\right]\right)$. Algorithm 5.3 gives

(1) $\left(a_{1}, b_{1}\right)=(131,50) \rightarrow(81,50) \rightarrow(31,50) \rightarrow(31,19) \rightarrow(12,19)$,

(2) $\left(c_{1}, d_{1}\right)=(131,47) \rightarrow(84,47) \rightarrow(37,47) \rightarrow(37,10) \rightarrow(27,10)$,

(3) $\left(m_{1}, m_{2}\right)=(0,1) \rightarrow(1,1) \rightarrow(2,1) \rightarrow(2,3) \rightarrow(5,3)$.

(4) $m_{1}+m_{2}=8$

\section{REFERENCES}

[1] A. Bogomolny, Stern-Brocot Tree. Binary Encoding; see the website: www.cut-the-knot.org/blue/encoding.shtml.

[2] R. Graham, D. Knuth and O. Patashnik, Concrete Mathematics, 2nd edition, AddisonWesley, 1994. MR.1397498 (97d:68003)

[3] J. L. Ramírez Alfonsín, Complexity of the Frobenius problem, Combinatorica 16 (1) (1996), 143-147. MR1394516 (97d:11058)

[4] J. L. Ramírez Alfonsín, The diophantine Frobenius problem, Oxford Univ. Press. (Vol. 30) 2005. MR2260521(2007i:11052)

[5] J. C. Rosales, P. A. García-Sánchez, J. I. García-García and J. M. Urbano-Blanco, Proportionally modular diophantine inequalities, Journal of Number Theory 103 (2003), 281-294. MR2020273 (2004k:20127)

[6] J. C. Rosales, P. A. García-Sánchez and J. M. Urbano-Blanco, The set of solutions of a proportionally modular diophantine inequality, Journal of Number Theory 128 (2008), 453467.

[7] J. C. Rosales and P. Vasco, The smallest positive integer that is a solution of a proportionally modular diophantine inequality, to appear in Mathematical Inequalities \& Applications (2008), www.ele-math.com.

[8] J. J. Sylvester, Mathematical questions with their solutions, Educational Times 41 (1884), 21.

Departamento de Álgebra, Universidad de Granada, 18071 Granada, Spain

E-mail address: bullejos@ugr.es

Departamento de Álgebra, Universidad de Granada, 18071 Granada, Spain

E-mail address: jrosales@ugr.es 